\title{
Towards joint state estimation and control in minimax MPC
}

\author{
Johan Löfberg \\ Department of Electrical Engineering \\ Linköpings universitet, SE-581 83 Linköping, Sweden \\ WWW: http://www. control.isy.liu.se \\ Email: johanl@isy.liu.se
}

June 18, 2001

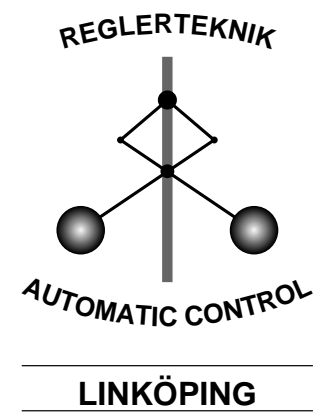

Report no.: LiTH-ISY-R-2357

Technical reports from the Automatic Control group in Linköping are available by anonymous ftp at the address ftp.control.isy.liu.se. This report is contained in the file 2357.pdf. 



\title{
Towards joint state estimation and control in minimax MPC
}

\author{
Johan Löfberg
}

June 18, 2001

\begin{abstract}
It is shown that joint deterministic state estimation and minimax MPC can be written as an optimization problem with linear and quadratic matrix inequalities. By linearizing the quadratic matrix inequality, a semidefinite program is obtained. A simulation study indicates that solving the joint problem can improve performance.
\end{abstract}

\section{Introduction}

The class of systems we address are linear discrete-time time-invariant systems with disturbed output measurements

$$
\begin{aligned}
x(k+1) & =A x(k)+B u(k) \\
y(k) & =C x(k)+\eta(k)
\end{aligned}
$$

The measurement error is assumed to be unknown but bounded

$$
\eta(k) \in\left\{\eta: \eta^{T} W \eta \leq 1\right\}, \quad W=W^{T} \succ 0
$$

The system analyzed in [Löf01] with external disturbances on the system dynamics can also be dealt with using the method proposed here, but for simplicity we restrict the case to measurement errors.

Since we only measure a disturbed output, we have to use a state estimator. Regardless of how this is done, we can write

$$
x(k)=\hat{x}(k)+e(k)
$$

The method that we will use here gives a state estimate with a guaranteed ellipsoidal error bound

$$
e^{T}(k) P(k) e(k) \leq 1
$$

The confidence matrix $P(k)$ is an output from the state estimation procedure. In the calculation of $\hat{x}(k)$ and $P(k)$ there is some degree of freedom. This note gives some ideas on how this can be exploited in order to improve closed loop performance when the estimate is used in the minimax MPC strategy proposed in [Löf01]. More precisely, it is shown that the joint state estimation problem and minimax MPC can be cast as an optimization problem involving a (unfortunately) quadratic matrix inequality. It is shown how this can be conservatively approximated as a linear matrix inequality (LMI) and thus enable us to approximately solve the joint problem using semidefinite programming. 


\section{Minimax MPC}

In MPC, the typical performance measure to minimize is a quadratic finite horizon $\left(Q=Q^{T} \succ 0, R=R^{T} \succ 0\right)$

$$
J=\sum_{j=0}^{N-1}\|x(k+j+1 \mid k)\|_{Q}^{2}+\|u(k+j \mid k)\|_{R}^{2}
$$

There are typically constraints on inputs and outputs, but to keep the notation simple, we will not write this explicitly. We will however return to the constraints later on. Exactly how the performance measure is used in MPC is beyond the scope of this note, see e.g. [Löf01] for details.

Since $x(k)$ is uncertain, this has to be addressed in some way. The standard approach to robustify nominal MPC is to employ a minimax strategy, i.e. optimize worst-case behavior [KBM94, BG97, SM98]. In [Löf01], it was shown that, given an ellipsoidal estimation error bound

$$
(x(k)-\hat{x}(k))^{T} P(k)(x(k)-\hat{x}(k)) \leq 1
$$

a minimax strategy

$$
\min _{u(\cdot)} \max _{x(k)} J
$$

gives a tractable problem that can be solved using semidefinite programming. The estimation part was performed without any consideration on how the estimate would influence the control performance. The results here extends those results and the goal is to connect the estimation part with the minimax controller.

\section{Deterministic state estimation}

So what is an optimal state estimate in a minimax framework? Clearly, the optimal choice is to find the smallest set $\mathcal{X}(k)$ such that

$$
x(k) \in \mathcal{X}(k)
$$

can be guaranteed, given all measurement obtained since startup and perhaps some prior knowledge on the initial state $x(0) \in \mathcal{X}(0)$. Hence the problem is

$$
\min \operatorname{Vol}(\mathcal{X}(k)) \text { given } y(1), y(2), \ldots y(k), \mathcal{X}(0)
$$

The crux is that this is not practically implementable, not even for our simplified model. The problem is that the complexity of the set $\mathcal{X}(k)$ grows when more measurements are obtained. The standard way to overcome this problem is to restrict $\mathcal{X}(k)$ to have some special geometry, such as ellipsoidal [Sch68, Sch73, GC99] or a parallelotope [BG97]. Furthermore, a recursive scheme is employed. Unfortunately, assuming that $\mathcal{X}(k-1)$ has some particular geometry does not imply that $\mathcal{X}(k)$ also will have this. Hence, if we force $\mathcal{X}(k)$ to be an ellipsoid, we will have to settle with an approximation. When we resort to an approximation, there will be some degree of freedom, and this is the fact we will exploit in order to improve the performance of the minimax MPC controller. 


\subsection{Ellipsoidal state estimates}

In this work, we will work with ellipsoidal approximations of the set $\mathcal{X}(k)$. Given a guaranteed ellipsoidal set for $x(k-1)$

$$
(x(k-1)-\hat{x}(k-1))^{T} P(k-1)(x(k-1)-\hat{x}(k-1)) \leq 1
$$

and a new measurement $y(k)$, use the model (1) and the measurement error bound (2) to find a new state estimate guaranteed to satisfy

$$
(x(k)-\hat{x}(k))^{T} P(k)(x(k)-\hat{x}(k)) \leq 1
$$

By defining the matrices

$$
\begin{aligned}
T_{e(k)} & =\left[\begin{array}{lll}
A & 0 & B u(k-1)-\hat{x}(k)
\end{array}\right] \\
T_{e(k-1)} & =\left[\begin{array}{lll}
I & 0 & -\hat{x}(k-1)
\end{array}\right] \\
T_{y} & =\left[\begin{array}{lll}
-C A & -I & y(k)-C B u(k-1)
\end{array}\right] \\
T_{\eta} & =\left[\begin{array}{lll}
0 & I & 0
\end{array}\right] \\
T_{1} & =\left[\begin{array}{lll}
0 & 0 & 1
\end{array}\right] \\
\Lambda & =T_{1}^{T} T_{1} \\
S_{e} & =\Lambda-T_{e(k-1)}^{T} P(k-1) T_{e(k-1)} \\
S_{y} & =\Lambda-T_{y}^{T} T_{y} \\
S_{\eta} & =\Lambda-T_{\eta}^{T} W T_{\eta}
\end{aligned}
$$

it can be shown that the following sufficient LMI is obtained after introducing the positive scalars $\tau_{e}$ and $\tau_{\eta}$ and the indefinite scalar $\tau_{y}$

$$
\left[\begin{array}{cc}
\Lambda-\tau_{e} S_{e}-\tau_{y} S_{y}-\tau_{\eta} S_{\eta} & T_{e(k)}^{T} \\
T_{e(k)} & P^{-1}(k)
\end{array}\right] \succeq 0
$$

The details are omitted, the reader is referred [GC99] or [Löf01] for the derivation. The important thing to notice is that the above expression is an LMI in the $\tau$ variables, $\hat{x}(k)$ and $P^{-1}(k)$.

Having this sufficient condition is a first step in a state estimation procedure. The next step is to select a particular solution $\hat{x}(k)$ and $P^{-1}(k)$. To do this, some performance measure on $P^{-1}(k)$ is minimized under the constraint (13). A typical choice [GC99] is trace $\left(P^{-1}(k)\right)$. We call this problem $\mathcal{P}_{1}$

$$
\begin{array}{rc}
\mathcal{P}_{1}: \min _{\tau, P^{-1}(k), \hat{x}(k)} & \operatorname{trace}\left(P^{-1}\right) \\
\text { subject to } & (13)
\end{array}
$$

However, if this is done, there is no connection to the control problem in which the state estimate will be used. The main result in this note is to show that the estimation, i.e. calculation of $\hat{x}(k)$ and $P(k)$, can be done simultaneously with the calculation of the control, thus leading to some sort of joint estimation and control. 


\section{The joint problem}

We first derive the LMI needed to solve the minimax problem. The calculations are done in a vectorized form so we introduce the predicted future states and control sequence

$$
X=\left[\begin{array}{c}
x(k+1 \mid) \\
x(k+2 \mid k) \\
\vdots \\
x(k+N \mid k)
\end{array}\right], U=\left[\begin{array}{c}
u(k \mid k) \\
u(k+1 \mid k) \\
\vdots \\
u(k+N-1 \mid k)
\end{array}\right]
$$

By introducing the matrices $H$ and $S$

$$
H=\left[\begin{array}{c}
A \\
A^{2} \\
\vdots \\
A^{N}
\end{array}\right], S=\left[\begin{array}{cccc}
B & 0 & \ldots & 0 \\
A B & B & \ldots & 0 \\
\vdots & \vdots & \ddots & \vdots \\
A^{N-1} B & A^{N-2} B & \ldots & B
\end{array}\right]
$$

we can write

$$
X=H x(k)+S U
$$

The minimax problem can, after redefining $Q:=\operatorname{diag}(Q, \ldots, Q)$ and $R:=\operatorname{diag}(R, \ldots, R)$, be written as

$$
\begin{array}{cc}
\min _{t, U} & t \\
\text { subject to } & \max _{x(k)} X^{T} Q X+U^{T} R U \leq t
\end{array}
$$

The state estimate uncertainty

$$
(x(k)-\hat{x}(k))^{T} P(k)(x(k)-\hat{x}(k)) \leq 1
$$

can be written in a form more suitable for us

$$
x(k)=\hat{x}(k)+P^{-1 / 2}(k) z,\|z\| \leq 1
$$

Rewrite the constraint in the minimax optimization problem using a Schur complement and introduce the uncertain state estimate

$$
\left[\begin{array}{ccc}
t & \hat{x}^{T}(k) H^{T}+z^{T} P^{-1 / 2} H^{T}+U^{T} S^{T} & U^{T} \\
H \hat{x}(k)+H P^{-1 / 2} z+S U & Q^{-1} & 0 \\
U & 0 & R^{-1}
\end{array}\right] \succeq 0
$$

Extract the uncertainty

$$
\begin{aligned}
& {\left[\begin{array}{ccc}
t & \hat{x}^{T}(k) H^{T}+U^{T} S^{T} & U^{T} \\
H \hat{x}(k)+S U & Q^{-1} & 0 \\
U & 0 & R^{-1}
\end{array}\right]} \\
& \quad+\left[\begin{array}{l}
I \\
0 \\
0
\end{array}\right] z^{T}\left[\begin{array}{lll}
0 & P^{-1 / 2} H^{T} & 0
\end{array}\right]+\left[\begin{array}{c}
0 \\
H P^{-1 / 2} \\
0
\end{array}\right] z\left[\begin{array}{lll}
I & 0 & 0
\end{array}\right] \succeq 0
\end{aligned}
$$

The above matrix inequality should hold for all admissible normalized estimation errors $z$. To proceed, we use the following theorem [GL97] 
Theorem 1 (Robust LMI) Robust satisfaction of the uncertain matrix inequality

$$
F+L \Delta R+R^{T} \Delta^{T} L^{T} \succeq 0 \quad \forall\|\Delta\| \leq 1
$$

is equivalent to the matrix inequality

$$
\begin{aligned}
{\left[\begin{array}{cc}
F & L \\
L^{T} & 0
\end{array}\right] } & \succeq\left[\begin{array}{ll}
R & 0 \\
0 & I
\end{array}\right]^{T}\left[\begin{array}{cc}
\tau I & 0 \\
0 & -\tau I
\end{array}\right]\left[\begin{array}{cc}
R & 0 \\
0 & I
\end{array}\right] \\
\tau & \geq 0
\end{aligned}
$$

After introducing the multiplier $\tau_{x} \geq 0$ and applying Theorem 1 to the uncertain LMI (19) we obtain

$$
\begin{aligned}
& {\left[\begin{array}{cccc}
t & \hat{x}^{T}(k) H^{T}+U^{T} S^{T} & U^{T} & 0 \\
H \hat{x}(k)+S U & Q^{-1} & 0 & H P^{-1 / 2} \\
U & 0 & R^{-1} & 0 \\
0 & P^{-1 / 2} H^{T} & 0 & 0
\end{array}\right] \succeq} \\
& {\left[\begin{array}{cc}
I & 0 \\
0 & 0 \\
0 & 0 \\
0 & I
\end{array}\right]\left[\begin{array}{cc}
\tau_{x} I & 0 \\
0 & -\tau_{x} I
\end{array}\right]\left[\begin{array}{cccc}
I & 0 & 0 & 0 \\
0 & 0 & 0 & I
\end{array}\right]}
\end{aligned}
$$

Simplification yields

$$
\left[\begin{array}{cccc}
t-\tau_{x} & \hat{x}^{T}(k) H^{T}+U^{T} S^{T} & U^{T} & 0 \\
H \hat{x}(k)+S U & Q^{-1} & 0 & H P^{-1 / 2} \\
U & 0 & R^{-1} & 0 \\
0 & P^{-1 / 2} H^{T} & 0 & \tau_{x} I
\end{array}\right] \succeq 0
$$

Given a state estimate, this is the LMI derived in [Löf01] to solve the minimax problem ${ }^{1}$. We denote this problem $\mathcal{P}_{2}$.

$$
\begin{array}{rc}
\mathcal{P}_{2}: & \min _{\tau_{x}, U, t} t \\
\text { subject to } & (21)
\end{array}
$$

We are now ready to proceed to the main idea in this work. Recall the state estimation LMI and introduce $Z=P^{-1 / 2}(k)$. The constraints for estimation and minimax MPC can be summarized as

$$
\begin{aligned}
& {\left[\begin{array}{cccc}
t-\tau_{x} & \hat{x}^{T}(k) H^{T}+U^{T} S^{T} & U^{T} & 0 \\
H \hat{x}(k)+S U & Q^{-1} & 0 & H Z \\
U & 0 & R^{-1} & 0 \\
0 & Z^{T} H^{T} & 0 & \tau_{x}
\end{array}\right] \succeq 0} \\
& {\left[\begin{array}{cc}
\Lambda-\tau_{e} S_{e}-\tau_{y} S_{y}-\tau_{\eta} S_{\eta} & T_{e(k)}^{T} \\
T_{e(k)} & Z^{T} Z
\end{array}\right] \succeq 0}
\end{aligned}
$$

Since $T_{e(k)}$ is linear in $\hat{x}(k)$, the equations are linear in the $\tau$ variables, $U$ and $\hat{x}(k)$. Unfortunately it is quadratic in $Z$. However, for future reference we define the problem as $\mathcal{P}_{3}$

$$
\mathcal{P}_{3}: \min _{\tau, Z, \hat{x}(k), U, t} t
$$

subject to

\footnotetext{
${ }^{1}$ Not entirely true. It is equivalent up to a similarity transformation
} 


\subsection{A tractable approximation}

To obtain a tractable problem, we linearize the quadratic matrix inequality. From the trivial inequality

$$
\left(Z-Z_{0}\right)^{T}\left(Z-Z_{0}\right) \succeq 0
$$

we have

$$
Z^{T} Z \succeq Z^{T} Z_{0}+Z_{0}^{T} Z-Z_{0}^{T} Z_{0}^{T}
$$

We use this and obtain an LMI that conservatively approximates the original quadratic matrix inequality

$$
\left[\begin{array}{cc}
\Lambda-\tau_{e} S_{e}-\tau_{y} S_{y}-\tau_{\eta} S_{\eta} & T_{e(k)}^{T} \\
T_{e(k)} & Z^{T} Z_{0}+Z_{0}^{T} Z-Z_{0}^{T} Z_{0}^{T}
\end{array}\right] \quad \succeq 0
$$

Clearly, the main problem now is to select the linearization point $Z_{0}$. The perhaps easiest solution is to solve the problem $\mathcal{P}_{1}$, and then use the solution to define $Z_{0}$. Of course, this can be repeated in order to find a local optimum of $\mathcal{P}_{3}$. We define the linearized and conservative approximation of $\mathcal{P}_{3}$

$$
\begin{array}{rc}
\mathcal{P}_{4}: & \min _{\tau, Z, \hat{x}(k), U, t} t \\
\text { subject to } & (22,26)
\end{array}
$$

\subsection{State constraints}

Typically there are state constraints in the MPC problem. Let us study the simple case $M X \leq 1$, where $M$ is a row vector. Inserting the definition of $X$ and the state estimate error yields the constraint

$$
M H \hat{x}(k)+M S U+M H P^{-1 / 2}(k) z \leq 1
$$

It is easy to show [Löf01] that the constraint is satisfied for all possible estimation errors if

$$
M H \hat{x}(k)+M S U+\sqrt{M H P^{-1}(k) H^{T} M^{T}} \leq 1
$$

Or equivalently

$$
\sqrt{M H P^{-1}(k) H^{T} M^{T}} \leq 1-M H \hat{x}(k)-M S U
$$

We square the constraint and recall $P^{-1}(k)=Z^{T} Z$. This allows us to perform a Schur complement and we obtain the LMI

$$
\left[\begin{array}{cc}
1-M H \hat{x}(k)-M S U & M H Z \\
Z^{T} H^{T} M^{T} & (1-M H \hat{x}(k)-M S U) I
\end{array}\right] \succeq 0
$$

\section{Simulation example}

This example is adapted from [BG00]. It is a second order poorly damped system

$$
\begin{aligned}
x(k+1) & =\left[\begin{array}{cc}
1.64 & -0.79 \\
1 & 0
\end{array}\right] x(k)+\left[\begin{array}{l}
1 \\
0
\end{array}\right] u(k) \\
y(k) & =\left[\begin{array}{ll}
0.14 & 0
\end{array}\right] x(k)+\eta(k)
\end{aligned}
$$


The measurement errors are guaranteed to be smaller than 0.2 hence

$$
W=25
$$

There is a non-minimum phase output $z(k)=\left[\begin{array}{ll}-1.93 & 2.21\end{array}\right] x(k)$ with a hard constraint

$$
-1 \leq z(k) \leq 3
$$

and the control has to satisfy $|u(k)| \leq 2$. The goal is to have the variable $y(k)$ track a constant unit reference, with performance weights $Q=C^{T} C$ and $R=0.1$. Since we have shifted the origin in this formulation, the following performance measure is used

$$
J=\sum_{j=0}^{N-1}\|C x(k+j+1 \mid k)-1\|^{2}+0.1\|u(k+j \mid k)-1\|^{2}
$$

The tracking formulation requires some modifications of the algorithm, but for brevity, the details are omitted. The horizon was $N=10$.

Three different controllers were implemented. In the first approach, the state estimation is performed by solving $\mathcal{P}_{1}$ and the estimate is then used in the minimax controller defined by $\mathcal{P}_{2}$. This is basically the controller proposed in [Löf01]. We denote this controller $\mathcal{C}_{1}$. In a second controller $\mathcal{C}_{2}$, an initial state estimate confidence matrix is found using $\mathcal{P}_{1}$, and the matrix is then used to linearize the joint problem $\mathcal{P}_{3}$, yielding $\mathcal{P}_{4}$ which then is solved. In a third approach $\mathcal{C}_{3}$, the linearization procedure is repeated two times.

The three controllers were simulated 100 times with different initial conditions and disturbance realizations. The initial state estimate was $\hat{x}(0)=0$ and $P(0)=I$, while the true initial state was uniformly distributed in the ellipsoid $x^{T}(0) x(0) \leq 1$. The measurement disturbances were uniformly distributed.

The mean of the accumulated quadratic performance measure,

$$
\sum_{j=0}^{\infty}\|C x(j)-1\|^{2}+0.1\|u(j)-1\|^{2}
$$

was calculated and was $J_{\mathcal{C}_{1}}=17.4, J_{\mathcal{C}_{2}}=10.4$ and $J_{\mathcal{C}_{3}}=8.8$. The average improvement when looking at single realizations and comparing the controllers $\mathcal{C}_{1}$ and $\mathcal{C}_{2}$ was $21 \%$, while $\mathcal{C}_{3}$ gave an additional $8 \%$ average improvement. Furthermore, the controller $\mathcal{C}_{1}$ became infeasible in 12 cases, while this never happened for $\mathcal{C}_{2}$ or $\mathcal{C}_{3}$. In Figure 1 , we see a situation were the proposed approach has improved tracking performance substantially.

The reason why the proposed approach gave such a substantial improvement in this example is the state constraint. The constrained output has a severe non-minimum phase behavior. If the uncertainty in the state estimate is too large, the uncertainty in the constrained output will force the controller to be very careful. Since the limiting factor is the constraint, it is important that the measurements are used in order to obtain an estimate that is certain along the constrained output directions. This will be done automatically in the joint approach, hence leading to improved performance. 


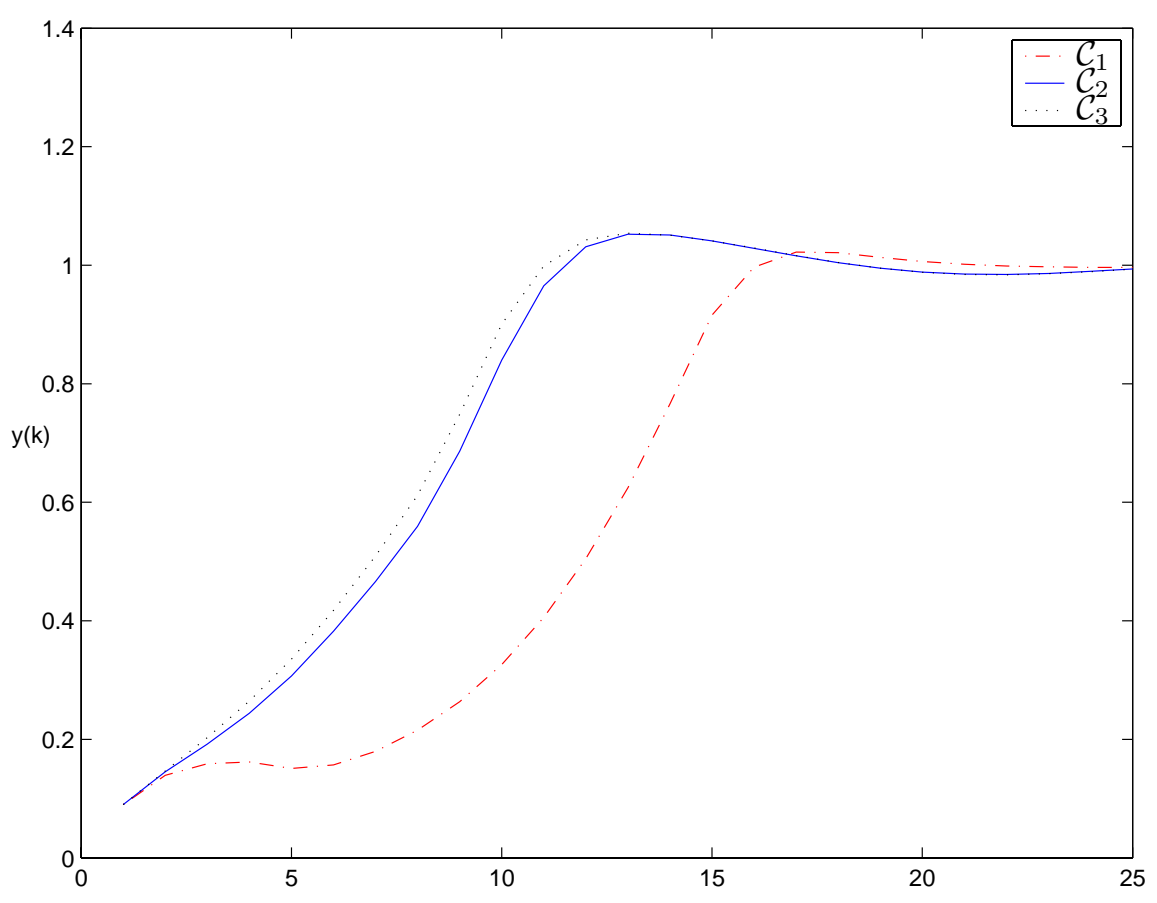

Figure 1: Closed loop response.

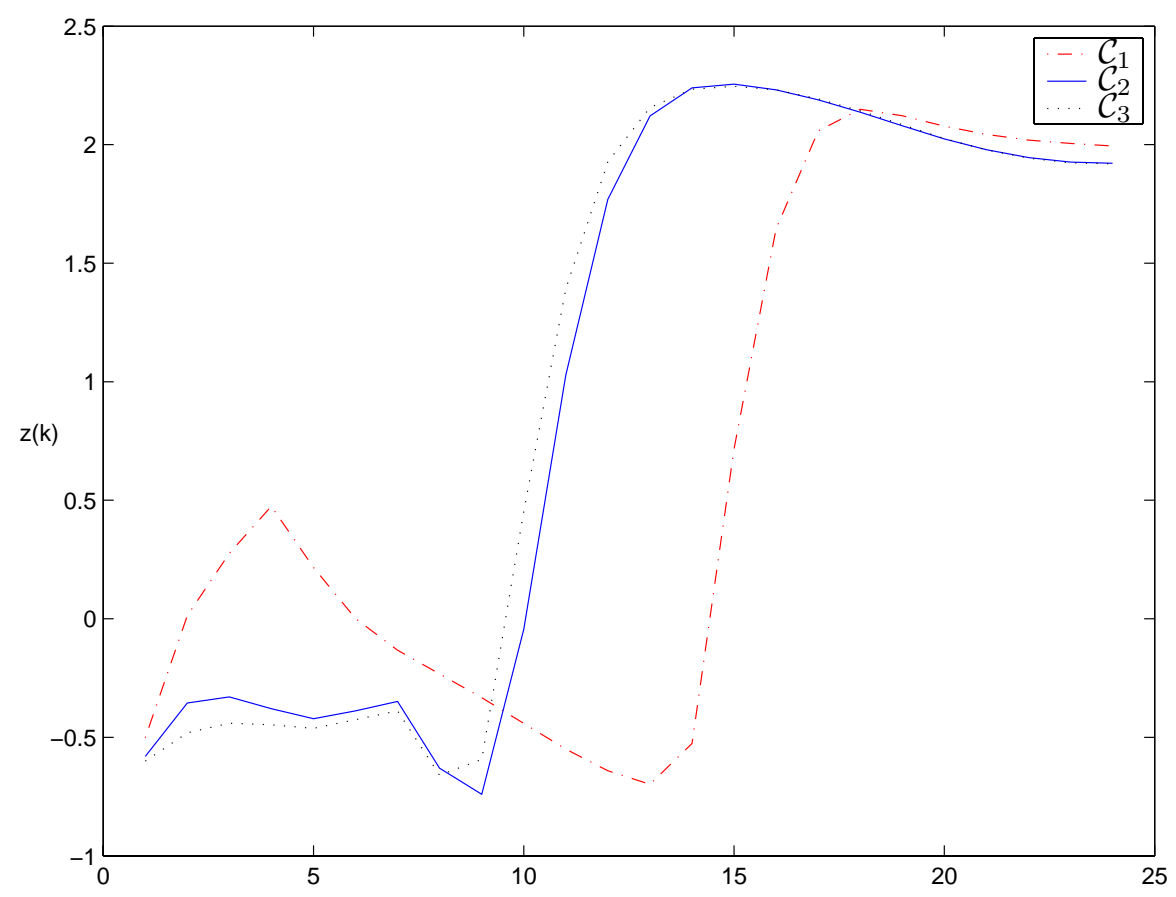

Figure 2: Constrained output. 


\section{Conclusion}

We have shown that incorporation of the state estimation problem into minimax MPC yields a problem with a quadratic matrix inequality. By linearizing this inequality, a linear matrix inequality is obtained, and the joint estimation and control problem could be solved using semidefinite programming. A simple simulation study was carried out and showed that the approach indeed can improve performance in some cases.

Of course, the improved performance comes at a price, computational complexity. Various improvements can be done to reduce this. Currently, the initial guess on $P(k)$ is found by solving problem $\mathcal{P}_{1}$. A cheaper way to find an initial guess could be to use the approximative solutions described in [Sch68, Sch73].

\section{References}

[BG97] A. Bemporad and A. Garulli. Predictive control via setmembership state estimation for constrained linear systems with disturbances. In Proceedings of the 4th European Control Conference, 1997.

[BG00] A. Bemporad and A. Garulli. Output-feedback predictive control of constrained linear systems via set-membership state estimation. International Journal of Control, 73(8):655-665, 2000.

[GC99] L. El Ghaoui and G. Calafiore. Worst-Case State Prediction under Structured Uncertainty. In Proceedings of the American Control Conference, pages 3402-3406, 1999.

[GL97] L. El Ghaoui and H. Lebret. Robust solutions to least-squares problems with uncertain data. SIAM Journal on Matrix Analysis and Applications, 18(4):1035-1064, 1997.

[KBM94] M.V. Kothare, V. Balakrishnan, and M. Morari. Robust constrained model predictive control using linear matrix inequilities. In Proceedings of the American Control Conference, pages 440-444, 1994.

[Löf01] J. Löfberg. Linear model predictive control: Stability and robustness. Licenciate thesis LIU-TEK-LIC-2001:03, Departement of Electrical Engineering, Linköpings universitet, Sweden, 2001.

[Sch68] F.C. Schweppe. Recursive state estimation: Unknown but bounded errors and system states. IEEE Transactions on Automatic Control, 13(1):22-28, February 1968.

[Sch73] F.C. Schweppe. Uncertain Dynamic Systems. Prentice-Hall, 1973.

[SM98] P.O.M. Scokaert and D.Q. Mayne. Min-max feedback model predictive control for constrained linear systems. IEEE Transactions on Automatic Control, 43(8):1136-1142, 1998. 